\title{
Implementation and Validation of a Fission Gas Release Model for CTFFuel using the NEA/OECD IFPE database
}

\author{
A. Abarca, M. Avramova, K. Ivanov \\ North Carolina State University, Raleigh, NC 27695 \\ aabarca@ncsu.edu,mnavramo@ncsu.edu,knivanov@ncsu.edu
}

\begin{abstract}
The nuclear reactors themselves are complex systems whose responses are driven by interactions between different physics phenomena within the reactor core. Traditionally, the different physics phenomena have been analyzed separately and its interaction considered via boundary conditions or closure models. However, in parallel with the development of computational technology, multi-physics coupled simulations are being used to obtain accurate predictions thanks to the consideration of the feedback effects on the fly (on-line). In the nuclear systems the fuel temperature is an important feedback parameter used to obtain the nuclear cross sections at given conditions by the neutron kinetics codes.
\end{abstract}

An accurate prediction of temperature profile within the fuel rod involve several physics such as neutron kinetics, mechanics, material behavior and properties, heat transfer, thermal-hydraulics, and even chemistry. The pellet to clad gap conductance is possibly the most important source of uncertainty in the solution of conductivity equation in the fuel rod and the fuel temperature prediction. The gap conductance depends on two effects: the pellet to gap distance and the conductivity of the gas species that fill the gap.

In this research work, the authors are focused on improving of the prediction of the gap gas conductivity in CTFFuel by implementing a fission gas release model in the code. The objective of this contribution is the implementation of a transient fission gas release model in CTFFuel and its validation using the experimental data available in the OECD/NEA International Fuel Performance Experiments (IFPE) database. CTFFuel is an isolated fuel heat transfer capability within the framework of CTF code, the state-of-theart version of the Coolant Boiling in Rod Arrays Code - Two-Fluid (COBRA-TF) subchannel thermal-hydraulic code. The code is being jointly developed by North Carolina State University (NCSU) and Oak Ridge National Laboratory (ORNL) within the US Department of Energy (DOE) Consortium for Advanced Simulation of LWRs (CASL).

KEYWORDS: CTFFuel, Fission Gas Release model, Implementation, Validation.

\section{INTRODUCTION}

An optimal fuel solver has to be designed to provide fuel temperature feedback with sufficient accuracy and low computational cost. With this premise as objective, CTFFuel was born as an isolated fuel heat transfer capability within the framework of CTF code, the modern version of the Coolant Boiling in Rod Arrays Code - Two-Fluid (COBRA-TF) sub-channel thermal-hydraulic code [1,2]. The code is being jointly developed by North Carolina State University (NCSU) and Oak Ridge National Laboratory (ORNL) for stand-alone and coupled applications in the Consortium for Advanced Simulation of Light Water Reactors (CASL)-developed core simulator, VERA. CTFFuel is the core fuel solver library in CTF 
and also includes a separate executable interface that allows a user to model a single rod with no fluid solution. This provides benefits such as the easy development and code-to-code verification or standalone fuel behavior uncertainty quantification.

The physical process of production and release of fission gases depends on the irradiation and power history of given fuel rod, as well as on the transient power changes. The released gases degrade the thermal conductivity of the fuel rod filling gas and increase the fuel rod internal pressure, thus affecting the thermal and mechanical responses. A mechanistic Fission Gas Release (FGR) model based on the $\mathrm{UO}_{2}$ grain size, the concentration of fission gas in the grain boundaries and the diffusion of the fission gases throughout the fuel pellet has been implemented in CTFFuel. The amount of fission gas released and impact of this model on the temperature prediction have been analyzed by the comparison with available experimental data summarized in the OECD/NEA International Fuel Performance Experiments (IFPE) database.

The development presented here can be considered as an extension of the capabilities of the CTFFuel code. The developed FGR model is applied in the code to compute the total quantity of gas in moles present in the pellet-cladding gap and the fraction of each one of the gas species found in the gap. With the final goal of improving the gap conductance prediction, the amount of fission gas computed by the model is used to update the value of the gap gas pressure and the conductivity of the mix of gas species that fill the gap.

This manuscript is organized as follows. In section 2 the FGR model implemented in CTFFuel is presented including the sub-models and correlations that drive the different physical phenomenon of the fission gas release. In section 3 the Verification and Validation $(V \& V)$ results of the model are presented and analyzed. The amount of gas predicted by the implemented model is analyzed by comparison with some available experimental data in the OECD/NEA IFPE database. A selection of steady-state and power-ramp cases has been done to verify the correctness of the implemented model. Finally, the authors underline the main conclusions in section 4.

\section{FISSION GAS RELEASE MODEL}

The process of production and release of fission gases depends on several parameters such as the irradiation time and power history of the fuel rod. The fission gas species released to the gap degrade the thermal conductivity and increase the fuel rod internal pressure, thus affecting the thermal and mechanical responses. There is available a significant quantity of fission gas release models available in the literature. However, some of the most important are the quantitative model developed by the American Nuclear Society, ANS-5.4-1982 [3] and its update the ANS-5.4-2011 [4], and the kinetic model developed by Forsberg and Massih [5] and its modifications.

\subsection{Implemented mechanistic fission gas release model}

A mechanistic FGR model has been implemented in CTFFuel to predict the amount of fission gas species released from the fuel pellet to the available space between the fuel pellet stack and the cladding. This model is based on the concentration of fission gas available in the grains and grains boundaries of the micro-structure of the fuel pellet. The kinetics is mainly based on the Forsberg and Massih [5] model and can be applied to both steady-state and fast transients. The developed model has similarities with the FRAPFGR model, developed at Pacific Northwest National Laboratory (PNNL) to initialize the transient gas release model in FRAPTRAN [6]. 


\subsection{Details of the model implementation}

The model has been implemented in the dynamic gap conductance modeling option of CTF/CTFFuel. This option is common between CTF and CTFFuel, so the model is available in both codes. For simplicity, CTFFuel has been used to make the verification of the implemented model. In this subsection, the different equations and correlations used to build the different physical phenomena that drive the mechanistic FGR model are presented.

\subsubsection{Fission gas production}

The straightforward model presented in equation (1) for the change in the fission gas production rate at radial pellet ring $\Delta C_{F G, i}$ in the time interval $[t ; t+\Delta t]$ is adopted for the fission gas production. This equation is applied at each integration point (ring) within the fuel pellet using the radial power shape calculated by a neutron kinetics code or provided by the user as an input:

$$
\Delta C_{F G, i}=\frac{\beta_{f} \cdot Y_{F G}}{A_{p} \cdot A_{v}} \cdot \bar{P}_{i} \cdot V_{i} \cdot \Delta t,
$$

where $Y_{F G}$ is the fractional yield of fission gas atoms per fission, $\beta_{f}$ is the conversion factor from $\mathrm{W}$ to fission/s (assuming $200 \mathrm{MeV} /$ fission), $A_{p}$ is the cross-sectional area of the fuel pellet based on asmanufactured dimensions, $A_{v}$ is the Avogadro's number, $V_{i}$ is the integration body volume (fuel pellet ring volume), $\dot{P}_{i}$ is the time average local power at the integration point (pellet radial location $\mathrm{r}$ ) and $\Delta t$ is the irradiation time step.

\subsubsection{Grain growth model}

The developed fission gas release model accepts an input grain size that can be specified in the input of CTFFuel. The default value for the grain size in the code is 10 micrometers $(\mu \mathrm{m})$, size which corresponds with the observed equivalent values applying the Mean Lineal Intercept (MLI) methodology to $\mathrm{UO}_{2}$. With the goal to represent the grain growth during the irradiation of the fuel rod, the dynamic model proposed by Khoruzhii et al. [7] has been selected. This model takes into account additional retardation effect existing under irradiation that matches with the available experimental data. The grain growth rate is described by expression (2):

$$
\frac{d a}{d t}=K \cdot\left(\frac{1}{a}-\frac{1}{a_{\max }}-\frac{1}{a_{i r r}}\right)[\mu m / h],
$$

In the presented model, the grain growth, $d a / d t$, depends on the current grain size, $a$, and terms related the retarding or growing limiting forces and irradiation effects in the growth $a_{\max }$ and $a_{i r r}$ respectively. The kinematic constant for the grain growth rate that depends on the pellet ring integral temperature is presented in equation (3):

$$
K=5.24 \times 10^{7} \cdot e^{(-32100 / T)}[\mu m / h]
$$

The retarding force is expressed through limiting grain size represented by equation (4). Finally, the expression (5) was developed to include the effects of the fission rate on the growth of the ceramic fuel grain: 


$$
\begin{gathered}
a_{\max }=2.23 \times 10^{3} \cdot e^{(-7620 / T)}[\mu m], \\
a_{i r r}=\frac{F_{0} \cdot T_{0}}{F \cdot T} \cdot A-e^{(-E / T)}[\mu m],
\end{gathered}
$$

In equation (5) $F_{0}=50 \mathrm{MW} / \mathrm{t} U$ and $T_{0}=1400 \mathrm{~K}$ are characteristic values of fission rate and fuel temperature, chosen for making variables dimensionless, and the model parameters $\mathrm{A}$ and $\mathrm{E}$ should be found on the basis of available experimental data. Comparing the available experimental data with the model predictions the authors recommend the optimal fitting values of $A=326.5 \mu \mathrm{m}$ and $E=5620 \mathrm{~K}$.

\subsubsection{High Burnup Rim Thickness}

The formation of a High Burnup (HB) rim is observed in the outer edge of highly irradiated fuel rod pellets. The microstructure of this HB pellet ring has been characterized by microscopy as a structure with sub-micron grains and high porosity. These two characteristics of the HB rim have been included in the developed fission gas release model.

The size of the HB rim ( $\left.t_{H B, \text { rim }}\right)$ has been measured via optical microscopy by Manzel and Walker [8], and they propose equation (6) to obtain an approximation of the rim width. The same authors also propose a grain size (MLI) for this recrystallized region of $0.15 \mu \mathrm{m}[9,10]$ :

$$
t_{H B, r i m}=1.439 \times 10^{-6} \cdot B u_{i}^{4.427}[\mu m],
$$

where $B u_{i}$ is the pellet ring burnup in GWd/MTU. Moreover, with the objective of preserving a smooth transition between the grain size of the HB neighboring rings, a linear interpolation has been applied for the grain size in the interval $\left[t_{H B, \text { rim }} ; 3 \cdot t_{H B \text {,rim }}\right]$.

\subsubsection{Gas diffusion constant}

The diffusion constant in the original Forsberg-Massih model [5] is defined over three temperature ranges between $675 \mathrm{~K}$ and $1850 \mathrm{~K}$. For fuel temperatures above or below this range, the diffusion coefficient keeps constant at the value calculated in the lower and upper limit of the range. Then, no extrapolation procedures are applied. Equation (7) shows the implemented procedure to compute the diffusion coefficient $(D(T))$ depending on the fuel ring temperature.

$$
\begin{aligned}
& D(T)=1.15 \times 10^{-23}\left[\mathrm{~m} / \mathrm{s}^{2}\right] \quad T \leq 675 K \\
& D(T)=1.51 \times 10^{-17} \cdot e^{\left(\frac{-9508}{T}\right)}\left[\mathrm{m} / \mathrm{s}^{2}\right] \quad 675 K<T \leq 1381 K \\
& D(T)=1.14 \times 10^{-13} \cdot e^{\left(\frac{-22884}{T}\right)}\left[\mathrm{m} / \mathrm{s}^{2}\right] \quad 1381 K<T \leq 1650 K \\
& D(T)=7.14 \times 10^{-10} \cdot e^{\left(\frac{-34879}{T}\right)}\left[\mathrm{m} / \mathrm{s}^{2}\right] \quad 1650 K<T \leq 1850 K \\
& D(T)=4.63 \times 10^{-18}\left[\mathrm{~m} / \mathrm{s}^{2}\right] \quad T>1850 K
\end{aligned}
$$




\subsubsection{Fission gas release model}

The developed model assumes that the fission gas produced in the fuel rod pellet diffuses to the available cladding gas expansion space depending on the gas concentration on the grain and in the grain boundaries. The diffusion process depends on the fuel temperature and the grain size. Regarding the grain size dependence, the FGR calculations are performed separately for recrystallized grains and nonrecrystallized grains. For pellet rings that contain both type grains, the releases from each type are combined based on the relative amount of each type of grain whereas the temperature dependence is considered in the calculation of the gas diffusion coefficient.

Physically, for the recrystallized grains, it is observed that the grains are so small (tens of a micron), that all of the produced gas diffuses out to the grain boundary. Therefore, the only gas that will remain in these grains at the end of given irradiation time step is the gas that is re-solved back into the grains. This resolved back fraction is given by the resolution factor from the Forsberg and Massih model [5] presented in equation (8):

$$
f_{\text {re-solved }}=\frac{1.81 \times 10^{-14} \cdot a_{\text {recry }}}{3 \cdot D(T)}
$$

The recrystallized grain size, $a_{\text {recry }}$, is equal to $0.075 \times 10^{-6} \mathrm{~m}$. Then, the gas that remains in the grain $G G_{i}^{n+1}$ of certain pellet ring, $i$, for the current irradiation step, $n+1$, is given by equation (9):

$$
G G_{i}^{n+1}=G B_{i}^{n}+\frac{1}{1-f_{\text {re-solved }}}\left[\mathrm{mol} / \mathrm{cm}^{3}\right]
$$

where $G B_{i}^{n}$ is the gas concentration in moles $/ \mathrm{cm}^{3}$ in the grain boundary of given pellet ring, $i$, for a the previous irradiation step, $n$.

For the non-recrystallized grains, the same formulas Forsberg-Massih model [5] are selected to calculate diffusion from the grains except that the gas release fraction is reduced to account for the effects of the gas resolution during the calculation of the released quantity. The Forsberg-Massih model is not fully described in this manuscript but can be found by the reader in [5].

The solution of the Forsberg-Massih gas diffusion equation to obtain the variation of gas concentration in the grain during given irradiation step, assuming constant production rate during the step and the possibility of resolution of gas from the grain boundary to the grain, can be written as equation (10):

$$
\Delta G_{n}=\frac{f_{n} \cdot G_{n}\left(\tau_{1}\right)}{\text { resolution }}+A_{n} \cdot \int_{\tau_{1}}^{\tau_{2}} \exp \left(\frac{-B_{n}}{a^{2}} \cdot\left(\tau_{2}-\tau_{0}\right) \cdot q\left(\tau_{0}\right) \cdot d \tau_{0}\right) \quad\left[\mathrm{mol} / \mathrm{cm}^{3}\right],
$$

The constants $A_{n}$ and $B_{n}$ (for $n=1, \ldots, 4$ ) are available in [5]. The total change of the gas concentration in the grain is given by equation (11):

$$
\Delta G G_{i}=\sum_{n=1}^{4} \Delta G_{n, i} \quad\left[\mathrm{~mol} / \mathrm{cm}^{3}\right]
$$


In the same way, the concentration of fission gas in the grain boundary is given by equation (12) in which the summation is directly applied:

$$
\Delta G B_{i}=\sum_{n=1}^{4}\left(f_{n} \cdot G_{n, i}\left(\tau_{1}\right)+A_{n} \cdot \int_{\tau_{1}}^{\tau_{2}} g\left(\tau_{2}-\tau_{0}\right) \cdot q\left(\tau_{0}\right) \cdot d \tau_{0}\right) \quad\left[\mathrm{mol} / \mathrm{cm}^{3}\right],
$$

In equations (10) and (12) the term $f_{n}$ represents the fission gas production fraction remaining in the grain from the previous time step, given by expression (13) and kinetic function $q(\tau)$ is obtained solving equation (14):

$$
\begin{gathered}
f_{n}=\exp \left(\frac{-B_{n} \cdot\left(\tau_{2}-\tau_{0}\right)}{a^{2}}\right)-1, \\
\frac{a^{2} \cdot q \cdot\left[-\sum_{n=1}^{4}\left(\frac{f_{n} \cdot A_{n}}{B_{n}}\right)+g(\Delta \tau)\right]}{\text { resolution }}=\beta \cdot \Delta t,
\end{gathered}
$$

where $a$ is the grain size, the constants $A_{n}$ and $B_{n}$ (for $\left.n=1, \ldots, 4\right)$ and $\beta$, and the function $g(\Delta \tau)$ are available in [5]. The resolution term present in equations (10) and (14) only depends on the fuel temperature and is obtained by applying equation (15):

$$
\begin{aligned}
& \max \left(1.0 ; 0.14 \cdot e^{0.00282 \cdot T}\right) \quad T \leq 1528.77 K \\
& \max (1.0 ; 22.976-0.0082 \cdot T) \quad T>1528.77 K
\end{aligned},
$$

Finally, in order to release fission gas from the grain boundaries, the saturation concentration must be reached. The saturation concentration in the grain boundaries is calculated using equation (16):

$$
G S_{i}=\frac{3 \cdot N S_{i}}{2 \cdot a}\left[\mathrm{~mol} / \mathrm{cm}^{3}\right]
$$

In equation (16), $N S_{i}$ is the saturation area density in moles $/ \mathrm{cm}^{3}$. This quantity can be calculated using the correlation proposed by Forsberg-Massih in [5].

The adopted criterion for the gas release is drove by the gas concentration in the grain boundary. When the gas concentration in the grain boundary for a given radial ring exceeds the saturation value for the first time, all the gas on the grain boundary except 65 percent of the saturation value is released. From then on for that ring, any gas above 65 percent of the saturation values is released. This criterion is applicable for both steady-state and fast transients. However, for the utilization of this model in fast transients, the model has to depart from given grain and boundary grain fission gas concentrations as well as a flag to indicate whether the saturation concentration in the grain boundary was reached or not during the irradiation time in given radial ring of the pellet is introduced.

\section{RESULTS AND DISCUSSION}

In this section Verification and Validation $(\mathrm{V} \& \mathrm{~V})$ of the implemented FGR model is presented. To perform this $\mathrm{V} \& \mathrm{~V}$ exercise, the amount of gas predicted is compared with some of the available 
OECD/NEA IFPE database experimental data. Besides this validation against the experimental data, due to the similarities between the implemented model and the modified Forsberg-Massih coded in FRAPCON, a code-to-code verification has been done.

Steady-state and power-ramp test cases have been selected from the available IFPE database experimental data to verify the correctness of the implemented model. In the presented analysis, uncertainties are not taken from experimental data (geometrical data, boundary conditions and FGR measurements) neither are propagated though the model during the simulation so that the authors are not able to provide in this study a comparison including the measurements and predictions confidence intervals. The obtained results are presented separately in two different subsections.

\subsection{Steady-state $V \& V$ cases}

The model accuracy to predict $\mathrm{FGR}$ in $\mathrm{UO}_{2}$ fuel rods has been assessed based on comparisons to experimental data and code-to-code comparison in 4 fuel rods with different power histories. The selected tests have a relatively constant power during the irradiation life so they can be considered as steady-state cases. Table I contains FGR comparison. In general, a slightly FGR overprediction is observed between the measured FGR and the predicted by the model.

Table I: Steady-State UO 2 FGR Verification Cases.

\begin{tabular}{|c|c|c|c|c|}
\hline Case & $\begin{array}{c}\text { Rod-average } \\
\text { burnup } \\
\text { (GWd/TMU) }\end{array}$ & $\begin{array}{c}\text { Measured FGR } \\
(\%)\end{array}$ & $\begin{array}{c}\text { FRAPCON-4.0* } \\
\text { predicted FGR } \\
(\%)\end{array}$ & $\begin{array}{c}\text { CTFFuel predicted } \\
\text { FGR (\%) }\end{array}$ \\
\hline IFA-597-3 & 70.34 & 15.80 & 14.55 & 20.91 \\
\hline $28 \mathrm{i} 6$ & 53.30 & 13.20 & 13.44 & 15.40 \\
\hline $36 \mathrm{i} 8$ & 61.50 & 33.80 & 38.09 & 41.37 \\
\hline $24 \mathrm{i} 6$ & 60.10 & 21.80 & 22.70 & 22.82 \\
\hline
\end{tabular}

\subsection{Power-rampV\&V cases}

The capability of the implemented model to predict FGR in $\mathrm{UO}_{2}$ fuel rods with power bumping (increase in rod power) at the End Of Life (EOL) is verified through another 4 tests. The code-to-code comparisons and the agreement of the prediction with the experimental data is presented in Table II. The FGR values in these power-ramp V\&V cases are overpredicted by CTFFuel.

Table II: Power-ramp FGR V\&V Cases.

\begin{tabular}{|c|c|c|c|c|c|}
\hline Case & $\begin{array}{c}\text { Power } \\
\text { ramp time } \\
(\mathrm{h})\end{array}$ & $\begin{array}{c}\text { Rod-average } \\
\text { burnup } \\
(\mathrm{GWd} / \mathrm{TMU})\end{array}$ & $\begin{array}{c}\text { Measured FGR } \\
(\%)\end{array}$ & $\begin{array}{c}\text { FRAPCON-4.0* } \\
\text { predicted FGR } \\
(\%)\end{array}$ & $\begin{array}{c}\text { CTFFuel predicted } \\
\text { FGR (\%) }\end{array}$ \\
\hline Risø ge2 & 70 & 41.90 & 24.60 & 25.64 & 29.57 \\
\hline Risø ge4 & 70 & 23.96 & 27.00 & 18.31 & 30.19 \\
\hline Risø AN1 & 70 & 41.30 & 34.16 & 25.54 & 40.78 \\
\hline Risø AN8 & 20 & 40.30 & 13.85 & 5.47 & 8.84 \\
\hline
\end{tabular}

*Using the modified Forsber-Massih model as FGR option. 


\section{CONCLUSIONS}

An optimal fuel solver must provide accurate fuel temperature feedback prediction with low computational cost. With this premise as objective, CTFFuel was born as an isolated fuel heat transfer capability within the framework of CTF code. A new mechanistic FGR model has been implemented in CTFFuel to predict the amount of fission gas isotopes released to the gap with the objective of improve the accuracy of the gap conductance predictions.

The developed FGR model is based on the concentration of fission gas available in the grain and grain boundaries of the micro-structure of the fuel pellet as well as the boundary grain saturation concentration. The kinetics is mainly based on the Forsberg and Massih [5] model and can be applied to both steadystate irradiations and fast transients.

The results presented in this manuscript show in general good agreement between the implemented model and the experimental data. Additionally, the code-to-code comparison against the modified Forsber and Massih model of FRAPCON has been used as a verification of the correctness of the FGR model implementation in CTFFuel. The results for the steady-state irradiation test are in good agreement with the experimental data whereas overall results for the power ramp V\&V cases are overpredicted by the model. The large experimental uncertainties are one of the major contributors to the discrepancies observed between the code prediction and the measured value.

Further V\&V activities of FGR model including the experimental uncertainties and the propagation of this uncertainties though the model are planned as a future lines of the current research project.

\section{REFERENCES}

1. M. Avramova, et. al, "CTF User's Manual", Version 4.0, (2019).

2. A. Toptan, R.Salko, M. Avramova, "CTFFuel User's Manual”, (2019).

3. American Nuclear Society (ANS). 1982. "Method for Calculating the Fractional Release of Volatile Fission Products from Oxide Fuel." ANS5.4 of the Standards Committee of the American Nuclear Society, ANSI/ANS-5.4-1982 (1982).

4. American Nuclear Society (ANS). 2011. "Method for Calculating the Fractional Release of Volatile Fission Products from Oxide Fuel." ANS5.4 of the Standards Committee of the American Nuclear Society, ANSI/ANS-5.4-2011 (2011).

5. Forsberg K and AR Massih. "Diffusion Theory of Fission Gas Migration in Irradiated Nuclear Fuel UO2." Journal of Nuclear Materials 135:140-148, (1985).

6. K. J. Geelhood et. al, "FRAPCON4.0 A Computer Code for the Calculation of Steady-State, ThermalMechanical Behavior of Oxide Fuel Rods for High Burnup" PNNL-19418, Vol.1 Rev.2, (2015).

7. Khoruzhii, O.V., S.Yu. Kourtchatov, and V.V. Likhanskii. "New model of equiaxed grain growth in irradiated UO2", Journal of Nuclear Materials, 265:112-116 (1999).

8. Manzel R and CT Walker. "High Burnup Fuel Microstructure and Its Effect on Fuel Rod Performance." LWR Fuel Performance Meeting, Park City, UT (2000).

9. Manzel R and CT Walker. "EPMA and SEM of fuel samples from PWR rods with an average burn-up of around $100 \mathrm{MWd} / \mathrm{kgHM}$." Journal of Nuclear Materials 301:170-182 (2002).

10. K. J. Geelhoo, W. G. Luscher, "FRAPCON-4.0: Integral Assessment" PNNL-19418, Vol.2 Rev.2, (2015). 